TITLE:

\title{
Swelling and deswelling kinetics of poly(N-isopropylacrylamide) gels
}

AUTHOR(S):

Takahashi, K; Takigawa, T; Masuda, T

\section{CITATION:}

Takahashi, K ...[et al]. Swelling and deswelling kinetics of poly( $\mathrm{N}$ iso propylacrylamide) gels. JOURNAL OF CHEMICAL PHYSICS 2004, 120(6): 2972-2979

\section{ISSUE DATE:}

2004-02-08

URL:

http://hdl.handle.net/2433/39756

\section{RIGHT:}

Copyright 2004 American Institute of Physics. This article may be downloaded for personal use only. Any other use requires prior permission of the author and the American Institute of Physics. 


\title{
Swelling and deswelling kinetics of poly $(\boldsymbol{N}$-isopropylacrylamide $)$ gels
}

\author{
Katsunori Takahashi, Toshikazu Takigawa, a) and Toshiro Masuda \\ Department of Material Chemistry, Kyoto University, Nishikyo-ku, Kyoto 615-8510, Japan
}

(Received 1 August 2003; accepted 3 November 2003)

\begin{abstract}
Swelling and deswelling kinetics was investigated for three types of cylindrical poly( $N$-isopropylacrylamide) (PNIPA) gels differing in crosslink density. The temperature dependence curves of the volume of the gel specimens were different from one another. One of the gel specimens was considered as a critical gel showing the continuous volume phase transition. The volume change process of the specimens after a temperature jump was examined. In the deswelling processes with temperature jumps to temperatures higher than $35^{\circ} \mathrm{C}$, a phase separation was observed in the gel specimens and the volume change slowed down due to the homogenization after the phase separation. The value of the diffusion constant obtained without the phase separation decreased rapidly as temperature approaches the transition temperature. The rapid decrease for the critical gel indicates the emergence of the critical slowing-down. The value of the critical exponent for the correlation length suggests that the universality class for the volume phase transition of the critical PNIPA gel belongs to the class for the classical theory. (C) 2004 American Institute of Physics. [DOI: 10.1063/1.1636695]
\end{abstract}

\section{INTRODUCTION}

A polymer gel is a three-dimensional polymer network swollen with solvent. The volume of the gel in solvent changes according to the change of an external field, such as temperature and $\mathrm{pH}$. This is because the change of the external field moves the thermodynamic equilibrium state of the gels. The gel in solvent changes the volume by swelling (or deswelling) until a new equilibrium state is achieved. Swelling and deswelling kinetics for polymer gels has been widely studied, and some theoretical approaches to the swelling kinetics has been made. ${ }^{1-5}$ These approaches are based on a kind of linear diffusion equation proposed by Tanaka et al. ${ }^{6}$ and are not applicable to large volume change processes. Matsuo et al. have studied swelling and deswelling kinetics in large volume change processes using a spherical gel, and proposed that the diffusion constant should be modified by introducing the concentration dependence into the diffusion constant. ${ }^{7}$ However, how to modify the diffusion constant is still unclear.

Poly( $N$-isopropylacrylamide) (PNIPA) gels are well known as typical of thermo-sensitive polymer gels, and show a steep volume change in a narrow temperature range of $\sim 35^{\circ} \mathrm{C}$. When crosslink density of the network is low enough, the PNIPA gel changes the volume discontinuously at a certain temperature around $35^{\circ} \mathrm{C}$. This is called the discontinuous volume phase transition of polymer gels. The degree of discontinuity at the transition temperature decreases with increasing crosslink density, and discontinuity is vanished when the crosslink density becomes high enough. The PNIPA gel at a certain crosslink density shows the continuous volume phase transition, where the slope of volume against temperature diverges at a certain temperature (critical

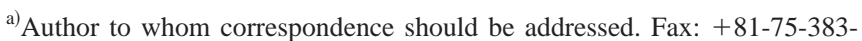
2458. Electronic mail: takigawa@rheogate.polym.kyoto-u.ac.jp
}

temperature). This continuous volume phase transition is regarded as the second-order phase transition and the gel showing the continuous transition is called a critical gel. The PNIPA gels having higher crosslink density than the critical gel change the volume continuously against temperature but remains finite over the whole temperature range. This means that no "volume phase transition" occurs for the gels in a thermodynamical sense. For the critical gels, physical quantities such as the correlation length and the specific heat show the critical behavior near the critical temperature. ${ }^{8-10}$ Tanaka et al. examined the critical kinetics of the PNIPA (critical) gel to show that the characteristic time of volume change diverges at the critical point. ${ }^{11}$ On the basis of these studies, ${ }^{8-11}$ the universality class of the volume phase transition was concluded to be identical to that for the threedimentional (3D) Ising model, but there appears to be no consensus for the universality class.

Recently, it has been shown that the volume phase transition in the course of deswelling (or shrinking) for the PNIPA gels becomes very slow, ${ }^{12-17}$ and the kinetics cannot be described by the conventional theory. ${ }^{1-7}$ This slow kinetics appears to be due to phase separation in the gels. We have also reported ${ }^{8}$ that the characteristic time for the deswelling is of the order of time needed for homogenization of the two phases. To explain the effects of phase separation on the kinetics, Shibayama et al. have proposed a mechanism for deswelling process by considering the inherent spatial inhomogeneity of the polymer gels. ${ }^{13,14}$ Suzuki et al. have investigated various phenomena observed in the deswelling process such as phase separation, bubble formation and phase coexistence, and they accounted for these phenomena on the basis of the classical phase separation models of nucleation and spinodal decomposition. ${ }^{15,16}$ For further understanding of the origin of the slow kinetics, detailed studies on how the phase separation affects the deswelling kinetics must be still needed. 
In this study, swelling as well as deswelling kinetics was examined for three types of PNIPA gels differing in crosslink density: One specimen shows the discontinuous phase transition and the second one shows a continuous volume change without phase transition, the final one being the critical gel. The effects of phase separation on the kinetics were investigated for various volume change processes induced by temperature jumps, and the kinetics for the three types of gels was compared with one another.

\section{EXPERIMENT}

Three types of cylindrical PNIPA gels with different crosslink density (coded hereafter as GEL1, GEL2, and GEL3) were prepared in distilled water by radical copolymerization of $N$-isopropylacrylamide (NIPA) as a main constituent and $N, N^{\prime}$-methylene-bis(acrylamide) (BIS) as a crosslink agent. Ammonium peroxodisulfate (AP) and $N, N$, $N^{\prime}, N^{\prime}$-tetramethylethylenediamine (TEMED) were used as an initiator and an accelerator, respectively. The monomer concentration (namely, the sum of the concentration of NIPA and BIS) was set to be $10.4 \mathrm{wt} \%$. The molar concentration ratio, [NIPA]/[BIS], was 20,60 , and 80 for GEL1, GEL2, and GEL3, respectively. The pregel solution was poured into glass tubes, in which the gelation was performed at $20^{\circ} \mathrm{C}$ for $24 \mathrm{~h}$. Then, the cylindrical PNIPA gels were removed from the glass tubes and immersed into the sufficient amount of distilled water to wash out the unreacted agent. The gels were cut into $15 \mathrm{~mm}$ long and used as specimens in measurements. The diameter of the specimens was $\sim 500 \mu \mathrm{m}$ in the equilibrium state at $20.0^{\circ} \mathrm{C}$.

Two types of measurements were employed in this study. One is the measurement in equilibrium (static measurement). The other is the measurement under the volume change (swelling and deswelling) process after a $T$-jump (dynamic measurement). In both measurements, size and appearances of the gel specimens were observed using a specially designed double-jacket water bath and a microscope equipped with a CCD camera. Temperature of the specimens $(T)$ was controlled by circulating the temperature controlled water in the outer jacket of the water bath. The accuracy in temperature was $\pm 0.1^{\circ} \mathrm{C}$ for the measurements.

First, the static measurement was performed. The $T$-dependence curve of the diameter of the specimens $(d)$ in the equilibrium state (the state where the diameter does not change with time at a fixed temperature in this experiment) was examined at various $T$ in a $T$-range of 20 to $80^{\circ} \mathrm{C}$. The measurement was performed on heating $\left(20^{\circ} \mathrm{C} \rightarrow 80^{\circ} \mathrm{C}\right)$ as well as on cooling $\left(80^{\circ} \mathrm{C} \rightarrow 20^{\circ} \mathrm{C}\right)$. The $T$-dependence curve of volume of the specimen $(V)$ was calculated by assuming that the swelling of the gel occurs isotropically; namely, by assuming

$$
V \propto d^{3} .
$$

After finishing the static measurement, the dynamic measurement was performed. The $T$-jump from the initial temperature $\left(T_{\mathrm{i}}\right)$ to the final temperature $\left(T_{\mathrm{f}}\right)$ was applied to the gel specimens, and the time $(t)$ dependence of $d$ was examined at a constant temperature of $T_{\mathrm{f}}$ until the volume approaches a constant value. In this study, the $T$-jump was

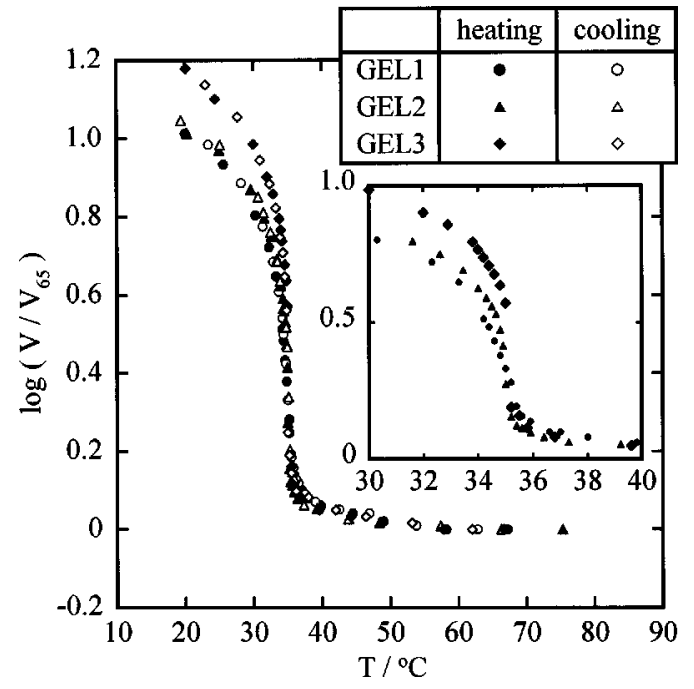

FIG. 1. Temperature $(T)$ dependence of normalized volume $V / V_{65}$ of three types of the PNIPA gels. Here, $V_{65}$ represents $V$ of each gel at $65{ }^{\circ} \mathrm{C}$. The inset shows the enlargement around $35^{\circ} \mathrm{C}$.

applied by transferring the gel specimens kept in water at $T_{\mathrm{i}}$ into the water controlled at $T_{\mathrm{f}}$, meaning that equilibrium of temperature in the gel specimens occurrs very shortly compared with the time scale of this experiment because the volume of water in the bath was $\sim 3 \mathrm{~cm}^{2}$ which is large enough compared with that of the specimens.

\section{RESULTS AND DISCUSSION}

As stated previously in the experimental section, the volume of the gel specimens in thermodynamic equilibrium state at each temperature $T$ was first examined. The volumetemperature $(V-T)$ curves measured on heating as well as on cooling for the three types of gels are shown in Fig. 1. In the equilibrium state, the gels were transparent regardless of temperature for the three gels. The volume used here is a normalized quantity calculated by using the volume of each specimen in the thermodynamic equilibrium state at $65.0^{\circ} \mathrm{C}\left(V_{65}\right)$. The volume decreases with increasing $T$, and shows a very steep change around $35^{\circ} \mathrm{C}$ for all kinds of specimens. The gels are in the swollen state in the $T$-range lower than $35^{\circ} \mathrm{C}$, and in the collapsed state at $T$ higher than $35^{\circ} \mathrm{C}$. The inset in Fig. 1 shows the magnified $V$ - $T$ curves on heating around $35^{\circ} \mathrm{C}$. As can be seen from the figure, the gel with the lowest crosslink density (GEL3) changes $V$ discontinuously at $35^{\circ} \mathrm{C}$, evidencing the discontinuous volume phase transition. The gel with the highest crosslink density (GEL1) changes $V$ continuously with $T$, showing no volume phase transition (of course, the volume change occurs for the gel). The gel with the intermediate crosslink density (GEL2) shows a very steep but continuous volume change around $35^{\circ} \mathrm{C}$, so that we regard hereafter the gel (GEL2) as a critical gel which shows the continuous volume phase transition. The $V$ - $T$ curve for GEL3 shows a hysteresis, which corresponds to $0.2^{\circ} \mathrm{C}$ in the transition temperature; namely, the transition occurs at $35.1{ }^{\circ} \mathrm{C}$ on heating, while at $34.9{ }^{\circ} \mathrm{C}$ on cooling. The transition temperature $\left(T_{\text {tr }}\right)$ for GEL3 was determined to be $35.0^{\circ} \mathrm{C}$ by averaging the two temperatures. 
For GEL1 and GEL2, no hysteresis was observed on the $V-T$ curves and even for these gels $T_{\text {tr }}$ was determined to be $35.0^{\circ} \mathrm{C}$ where the absolute value of slope for the curve holds a maximum.

The dynamic measurement was performed by the $T$-jump method for the three types of specimens. The values of $T_{\mathrm{i}}$ and $T_{\mathrm{f}}$ are summarized in Table I. The appearance of the gel specimens during the volume change process was different depending on the condition of the $T$-jump. In the deswelling (shrinking) processes at $T_{\mathrm{f}}$ higher than $35.0^{\circ} \mathrm{C}$, however, the opaqueness was observed for the three gels. The opaqueness must originate from the inhomogeneity of polymer concentration in the whole gel induced by the phase separation $^{12}$ and the phase separation temperature lay around $35.0^{\circ} \mathrm{C}$ regardless of the gel type. In other cases than the deswelling processes at $T_{\mathrm{f}}>35^{\circ} \mathrm{C}$ (namely, in the deswelling processes at $T_{\mathrm{f}}<35.0^{\circ} \mathrm{C}$ or the swelling processes), however, the gels changed $V$ without the phase separation (i.e., with keeping transparency).

For example, the $t$-dependence curve of $d$ for GEL2 after the $T$-jump from $T_{\mathrm{i}}=20.0^{\circ} \mathrm{C}$ to $T_{\mathrm{f}}=50.0^{\circ} \mathrm{C}$ is shown in Fig. 2. Since this is the deswelling process at $T_{\mathrm{f}}>35.0^{\circ} \mathrm{C}$, the gel specimen became opaque after the $T$-jump. As can be seen from the figure, $d$ decreases steeply just after the $T$-jump. In this time period, a bamboolike pattern was observed on the surface of the gel specimen. The gel with this large-scale surface pattern was composed of the transparent and opaque regions, the latter corresponding to the node of the bamboo. The node extended in the axial direction with increasing time, and finally the gel became fully opaque at about $30 \mathrm{~min}$ after the $T$-jump. This means that the second filled circle from the left in the figure corresponds to $d$ for fully opaque gel. This large-scale surface pattern was observed only in the deswelling processes at $T_{\mathrm{f}}>35.0^{\circ} \mathrm{C}$ with relatively large volume change.

The diameter of the opaque region $\left(d_{\text {in }}\right)$ is also plotted against $t$ in Fig. 2. The value of $d_{\text {in }}$ also decreases with $t$, and the decrease of $d_{\text {in }}$ corresponds to the extension of the transparent region from the surface to the center of the gel. As is seen from the figure, at $t=9.7 \times 10^{4} \mathrm{~s} d$ reaches an equilibrium value $\left(d_{\mathrm{f}}\right)$ and the opaque region disappears. The opaque gel specimen in a two-phase state, polymer rich and solvent rich phases, shrank with the progress of homogenization and the gel finally recovered the transparency at long time limit.

In order to determine the characteristic time $(\tau)$ for the change of $d$, it is convenient to use two parameters ( $\Delta d$ and $\Delta d_{0}$ ) defined by the following equations:

$$
\begin{aligned}
& \Delta d=d-d_{\mathrm{f}}, \\
& \Delta d_{0}=d_{\mathrm{i}}-d_{\mathrm{f}} .
\end{aligned}
$$

Here, $d_{\mathrm{i}}$ represents the diameter at $T_{\mathrm{i}}$. Figure 3 shows a semilogarithmic plot of $\Delta d / \Delta d_{0}$ against $t$ of the deswelling process after the $T$-jump from $T_{\mathrm{i}}=20.0^{\circ} \mathrm{C}$ to $T_{\mathrm{f}}=50.0^{\circ} \mathrm{C}$ for GEL2. The data here correspond to those in Fig. 2. The slope of the line gives the value of $\tau$ of $2.7 \times 10^{4} \mathrm{~s}$. As easily understood, $\tau$ estimated in this method corresponds to a longest relaxation time for the diameter, or volume, change. In the short time region of the deswelling, a large-scale surface pattern occurred for this specimen, but the longest relaxation time $\tau$ is not affected by the pattern formation because the time domains are quite different. When $\tau$ and $d_{\mathrm{f}}$ are determined, we can estimate the diffusion constant $(D)$ by using the following relation for cylindrical gels: ${ }^{18}$

$$
D=\frac{d_{\mathrm{f}}^{2}}{24 \tau} .
$$

The values of $D$ obtained for GEL2, is plotted against $T_{\mathrm{f}}$ in Fig. 4. Figure 4(a) shows the data for $T_{\mathrm{f}}<T_{\mathrm{tr}}\left(=35.0^{\circ} \mathrm{C}\right)$, and Fig. 4(b) for $T_{\mathrm{f}}>T_{\mathrm{tr}}$. Open and filled symbols represent the data on swelling and deswelling, respectively. The details are summarized in Table I, together with the data for GEL1 and GEL3. It is clear from the figure that $D$ in Fig. 4(b) depends on the total amount of volume change, or a swelling strain $\left(\gamma_{\mathrm{v}}\right)$ defined by

$$
\gamma_{\mathrm{v}}=\frac{V_{\mathrm{i}}-V_{\mathrm{f}}}{V_{\mathrm{f}}} .
$$

Here, $V_{\mathrm{i}}$ and $V_{\mathrm{f}}$ are the volumes at $T_{\mathrm{i}}$ and $T_{\mathrm{f}}$, respectively. The diffusion constant $D$ in Fig. 4(a) is independent of $\gamma_{\mathrm{v}}$ and the $T_{\mathrm{f}}$-dependence of $D$ on swelling and deswelling appears identical. On the other hand, $D$ on deswelling in Fig. 4(b) appears to decreases with increasing $\gamma_{\mathrm{v}}$ if comparison is made at a fixed temperature. The data points in Fig. 4(b) can be classified into several categories (four for deswelling and two for swelling) depending on the value of $\gamma_{\mathrm{v}}$. Of course, the grouping is an expedient and no clear border between neighboring groups exists. The values of $D$ for the deswelling process where the phase separation was observed are plotted against $\gamma_{\mathrm{v}}$ in Fig. 5. The values are very small and are almost independent of $\gamma_{\mathrm{v}}$ at $\gamma_{\mathrm{v}}>3$ where the large-scale surface pattern was observed. The homogenization after the phase separation requires long time and also moves the time needed for thermodynamic equilibration in dimension to the longer time side compared with that of the volume change process without the phase separation. As a result, the value of $D$ became small. On the other hand, $D$ increases rapidly with decreasing $\gamma_{\mathrm{v}}$ in the range of $\gamma_{\mathrm{v}}<2$. The phase separation was also observed in this $\gamma_{\mathrm{v}}$-range but the degree of opaqueness was relatively small compared with the case of $\gamma_{\mathrm{v}}>3$. This may give a shorter time for homogenization of the phase separation, resulting in a larger value of $D$. As can be seen in Fig. 4, it is clear that the effects of the homogenization after phase separation on the deswelling kinetics become negligible in the small $\gamma_{\mathrm{v}}$ limit, where $D$ in the deswelling process approaches to that in the swelling process.

The data points for $T_{\mathrm{f}}<T_{\text {tr }}$ in Fig. 4(a) appear to fall on a single curve, and the curve shows a steep decrease as $T_{\mathrm{f}}$ approaches $T_{\text {tr }}$, corresponding to the critical slowingdown. Although all data points for $T_{\mathrm{f}}>T_{\mathrm{tr}}$ in Fig. 4(b) cannot be approximated by a single curve due to the strong $\gamma_{\mathrm{v}}$-dependence of $D$, the data points marked by open symbols, which correspond to the data on swelling and are therefore free from the effects of the phase separation, might fall on a single curve, just as a mirror image of the curve expected for the data points in Fig. 4(a). In addition, seeing the 
TABLE I. Sample code, the final temperature $\left(T_{\mathrm{f}}\right)$, the initial temperature $\left(T_{\mathrm{i}}\right)$, the diffusion constant $(D)$, and the swelling strain $\left(\gamma_{\mathrm{v}}\right)$ of the dynamic $(T$-jump) measurements.

\begin{tabular}{|c|c|c|c|c|c|c|c|}
\hline & $T_{\mathrm{f}}\left({ }^{\circ} \mathrm{C}\right)$ & $T_{\mathrm{i}}\left({ }^{\circ} \mathrm{C}\right)$ & $D\left(\mathrm{~cm}^{2} \mathrm{~s}^{-1}\right)$ & $\gamma_{\mathrm{v}}$ & $\begin{array}{l}\text { Symbols } \\
\text { in Fig. } 7\end{array}$ & $\begin{array}{l}\text { Symbols } \\
\text { in Fig. } 9\end{array}$ & \\
\hline \multirow[t]{2}{*}{ GEL1 } & \begin{tabular}{|r|}
8.7 \\
15.4 \\
19.2 \\
27.6 \\
33.4 \\
33.6 \\
34.2 \\
35.0 \\
35.2 \\
35.4 \\
35.6 \\
35.9 \\
37.0 \\
37.0 \\
38.0 \\
39.0 \\
21.4 \\
28.2 \\
28.2 \\
32.0 \\
32.2 \\
33.6 \\
33.6 \\
34.3 \\
34.6 \\
34.8
\end{tabular} & \begin{tabular}{|l|}
20.0 \\
26.0 \\
27.6 \\
33.4 \\
34.2 \\
34.6 \\
34.8 \\
37.0 \\
39.8 \\
39.8 \\
45.0 \\
45.0 \\
45.0 \\
60.0 \\
60.0 \\
60.0 \\
8.0 \\
20.0 \\
20.3 \\
28.2 \\
28.2 \\
32.0 \\
32.2 \\
33.6 \\
33.6 \\
34.3
\end{tabular} & $\begin{array}{l}7.50 \times 10^{-7} \\
4.69 \times 10^{-7} \\
5.02 \times 10^{-7} \\
2.99 \times 10^{-7} \\
1.29 \times 10^{-7} \\
1.84 \times 10^{-7} \\
6.90 \times 10^{-8} \\
3.22 \times 10^{-8} \\
2.20 \times 10^{-8} \\
1.84 \times 10^{-8} \\
3.19 \times 10^{-8} \\
5.87 \times 10^{-8} \\
3.34 \times 10^{-8} \\
6.83 \times 10^{-8} \\
1.27 \times 10^{-7} \\
8.29 \times 10^{-8} \\
7.09 \times 10^{-7} \\
7.08 \times 10^{-7} \\
4.94 \times 10^{-7} \\
1.96 \times 10^{-7} \\
1.94 \times 10^{-7} \\
1.69 \times 10^{-7} \\
1.47 \times 10^{-7} \\
7.73 \times 10^{-8} \\
5.62 \times 10^{-8} \\
5.10 \times 10^{-8}\end{array}$ & $\begin{array}{l}-0.185 \\
-0.246 \\
-0.276 \\
-0.441 \\
-0.237 \\
-0.349 \\
-0.412 \\
-0.442 \\
-0.334 \\
-0.255 \\
-0.192 \\
-0.223 \\
-0.194 \\
-0.096 \\
-0.095 \\
-0.079 \\
0.346 \\
0.398 \\
0.339 \\
0.553 \\
0.504 \\
0.239 \\
0.215 \\
0.196 \\
0.505 \\
0.268\end{array}$ & $\begin{array}{l}0 \\
0 \\
0 \\
0 \\
0 \\
0 \\
0 \\
0 \\
\square \\
\square \\
\square \\
\square \\
\square \\
\square \\
\square \\
\square \\
0 \\
0 \\
0 \\
0 \\
0 \\
0 \\
0 \\
0 \\
0 \\
0 \\
0\end{array}$ & $\begin{array}{l}0 \\
0 \\
0 \\
0 \\
\square \\
\square \\
\square \\
\square \\
\square \\
\square \\
\square \\
\square \\
\square \\
0 \\
0 \\
0 \\
0 \\
0 \\
0 \\
0 \\
0 \\
0 \\
0\end{array}$ & \\
\hline & $T_{\mathrm{f}}\left({ }^{\circ} \mathrm{C}\right)$ & $T_{\mathrm{i}}\left({ }^{\circ} \mathrm{C}\right)$ & $D\left(\mathrm{~cm}^{2} \mathrm{~s}^{-1}\right)$ & $\gamma_{\mathrm{v}}$ & $\begin{array}{l}\text { Symbols } \\
\text { in Fig. 4(a) }\end{array}$ & $\begin{array}{c}\text { Symbols } \\
\text { in Fig. 4(b) }\end{array}$ & $\begin{array}{l}\text { Symbols } \\
\text { in Fig. } 6\end{array}$ \\
\hline GEL2 & $\begin{array}{r}5.6 \\
10.0 \\
15.0 \\
20.0 \\
28.7 \\
30.0 \\
32.0 \\
34.0 \\
20.0 \\
34.0 \\
36.1 \\
38.0 \\
40.0 \\
45.0 \\
35.4 \\
36.4 \\
35.5 \\
35.6 \\
35.9 \\
25.0 \\
28.7 \\
32.2 \\
33.9 \\
34.2 \\
34.4 \\
34.6 \\
45.0 \\
60.2 \\
37.0 \\
45.0 \\
45.6 \\
35.9 \\
36.5 \\
38.0 \\
40.0 \\
45.0 \\
45.0 \\
50.0 \\
50.4 \\
60.0 \\
60.2 \\
70.4 \\
40.0 \\
50.0 \\
60.0 \\
60.0 \\
60.0 \\
60.0\end{array}$ & $\begin{array}{l}20.0 \\
20.0 \\
25.0 \\
28.7 \\
32.0 \\
34.0 \\
34.0 \\
34.6 \\
60.0 \\
37.2 \\
45.0 \\
60.0 \\
60.0 \\
70.0 \\
40.0 \\
45.0 \\
45.0 \\
45.0 \\
45.0 \\
10.0 \\
20.0 \\
28.7 \\
32.2 \\
33.0 \\
33.0 \\
33.9 \\
43.0 \\
55.0 \\
35.9 \\
38.8 \\
41.7 \\
35.1 \\
35.3 \\
35.3 \\
35.3 \\
35.3 \\
35.3 \\
35.5 \\
35.5 \\
35.0 \\
36.0 \\
36.0 \\
20.0 \\
20.0 \\
20.0 \\
30.0 \\
33.0 \\
34.0\end{array}$ & $\begin{array}{l}6.32 \times 10^{-7} \\
4.59 \times 10^{-7} \\
5.78 \times 10^{-7} \\
4.08 \times 10^{-7} \\
3.09 \times 10^{-7} \\
2.12 \times 10^{-7} \\
1.83 \times 10^{-7} \\
1.22 \times 10^{-7} \\
4.21 \times 10^{-7} \\
7.85 \times 10^{-8} \\
4.17 \times 10^{-8} \\
5.67 \times 10^{-8} \\
3.38 \times 10^{-8} \\
1.39 \times 10^{-7} \\
1.37 \times 10^{-8} \\
5.56 \times 10^{-8} \\
2.11 \times 10^{-8} \\
2.55 \times 10^{-8} \\
3.24 \times 10^{-8} \\
3.76 \times 10^{-7} \\
3.34 \times 10^{-7} \\
1.65 \times 10^{-7} \\
9.86 \times 10^{-8} \\
9.75 \times 10^{-8} \\
5.36 \times 10^{-8} \\
2.29 \times 10^{-8} \\
1.07 \times 10^{-7} \\
1.19 \times 10^{-7} \\
6.55 \times 10^{-8} \\
2.46 \times 10^{-8} \\
1.94 \times 10^{-8} \\
1.40 \times 10^{-8} \\
2.42 \times 10^{-8} \\
7.41 \times 10^{-9} \\
5.28 \times 10^{-9} \\
3.42 \times 10^{-9} \\
3.98 \times 10^{-9} \\
5.52 \times 10^{-9} \\
1.06 \times 10^{-8} \\
4.21 \times 10^{-9} \\
7.38 \times 10^{-9} \\
9.23 \times 10^{-9} \\
7.52 \times 10^{-10} \\
7.36 \times 10^{-10} \\
1.11 \times 10^{-9} \\
1.02 \times 10^{-9} \\
1.14 \times 10^{-9} \\
1.40 \times 10^{-9}\end{array}$ & $\begin{array}{c}-0.199 \\
-0.213 \\
-0.270 \\
-0.290 \\
-0.334 \\
-0.394 \\
-0.257 \\
-0.320 \\
-0.931 \\
-0.709 \\
-0.088 \\
-0.088 \\
-0.067 \\
-0.036 \\
-0.192 \\
-0.103 \\
-0.175 \\
-0.175 \\
-0.119 \\
0.481 \\
0.500 \\
0.403 \\
0.467 \\
0.339 \\
0.548 \\
0.467 \\
0.028 \\
0.000^{\mathrm{a}} \\
0.037 \\
0.097 \\
0.037 \\
0.210 \\
0.132 \\
0.260 \\
0.456 \\
0.424 \\
0.397 \\
0.700 \\
0.422 \\
0.424 \\
0.256 \\
0.202 \\
10.305 \\
10.665 \\
13.414 \\
6.547 \\
5.985 \\
3.294\end{array}$ & $\begin{array}{l}0 \\
0 \\
0 \\
0 \\
0 \\
0 \\
0 \\
0 \\
0 \\
\diamond \\
\diamond\end{array}$ & 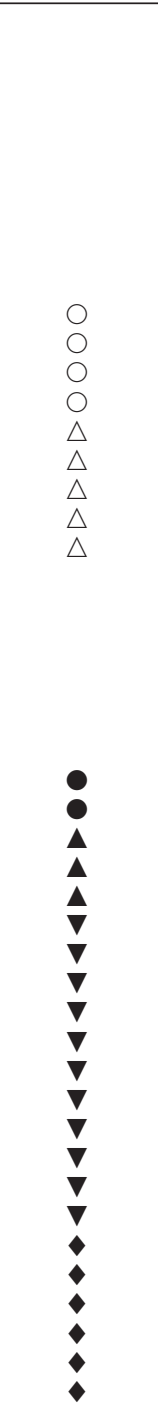 & $\begin{array}{l}0 \\
0 \\
0 \\
0 \\
0 \\
\\
\square \\
\square \\
\square \\
\square \\
\square \\
\square \\
\square \\
\square \\
\square \\
\square \\
0 \\
0 \\
0 \\
0 \\
0 \\
0 \\
0\end{array}$ \\
\hline
\end{tabular}


TABLE I. (Continued.)

\begin{tabular}{|c|c|c|c|c|c|c|}
\hline & $T_{\mathrm{f}}\left({ }^{\circ} \mathrm{C}\right)$ & $T_{\mathrm{i}}\left({ }^{\circ} \mathrm{C}\right)$ & $D\left(\mathrm{~cm}^{2} \mathrm{~s}^{-1}\right)$ & $\gamma_{\mathrm{v}}$ & $\begin{array}{l}\text { Symbols } \\
\text { in Fig. } 8\end{array}$ & $\begin{array}{l}\text { Symbols } \\
\text { in Fig. } 10\end{array}$ \\
\hline GEL3 & $\begin{array}{l}25.4 \\
28.8 \\
30.0 \\
31.9 \\
32.9 \\
33.4 \\
34.3 \\
34.6 \\
34.8 \\
34.8 \\
12.0 \\
20.2 \\
28.3 \\
31.6 \\
33.0 \\
35.4 \\
35.5 \\
35.7 \\
36.0 \\
37.0 \\
38.0\end{array}$ & $\begin{array}{l}12.0 \\
20.2 \\
25.4 \\
28.8 \\
30.0 \\
31.9 \\
33.4 \\
32.9 \\
33.4 \\
33.4 \\
20.2 \\
28.3 \\
31.6 \\
33.0 \\
34.2 \\
50.0 \\
50.0 \\
50.0 \\
50.0 \\
50.0 \\
50.0\end{array}$ & $\begin{array}{l}4.27 \times 10^{-7} \\
8.57 \times 10^{-7} \\
3.10 \times 10^{-7} \\
5.21 \times 10^{-7} \\
2.10 \times 10^{-7} \\
2.85 \times 10^{-7} \\
1.19 \times 10^{-7} \\
6.15 \times 10^{-8} \\
8.24 \times 10^{-8} \\
5.18 \times 10^{-8} \\
7.43 \times 10^{-7} \\
3.73 \times 10^{-7} \\
4.70 \times 10^{-7} \\
2.36 \times 10^{-7} \\
2.25 \times 10^{-7} \\
3.77 \times 10^{-8} \\
4.90 \times 10^{-8} \\
3.98 \times 10^{-8} \\
3.57 \times 10^{-8} \\
5.84 \times 10^{-8} \\
5.38 \times 10^{-8}\end{array}$ & $\begin{array}{l}0.310 \\
0.456 \\
0.434 \\
0.816 \\
0.352 \\
0.377 \\
0.344 \\
0.953 \\
0.559 \\
0.953 \\
-0.170 \\
-0.272 \\
-0.237 \\
-0.173 \\
-0.301 \\
-0.226 \\
-0.226 \\
-0.215 \\
-0.202 \\
-0.150 \\
-0.079\end{array}$ & $\begin{array}{l}0 \\
0 \\
0 \\
0 \\
0 \\
0 \\
0 \\
0 \\
0 \\
0 \\
0 \\
0 \\
0 \\
0 \\
0 \\
\square \\
\square \\
\square \\
\square \\
\square \\
\square\end{array}$ & $\begin{array}{l}0 \\
0 \\
0 \\
0 \\
0 \\
0 \\
0 \\
0 \\
0 \\
0 \\
0 \\
0 \\
0 \\
0 \\
\square \\
\square \\
\square \\
\square \\
\square \\
\square\end{array}$ \\
\hline
\end{tabular}

${ }^{a}$ In this deswelling process, the volume change was too small to detect, but $\mathrm{t} D$ was able to be estimated using the relation between $\tau$ and the time $\left(t_{\mathrm{d}}\right)$ needed for the homogenization after the phase separation $\left(t_{\mathrm{d}}=3.4 \tau\right)$ for the gel.

data on deswelling in $0<\gamma_{\mathrm{v}}<0.03$, the data points appear to fall on the same curve as that for the data points for swelling. This means that even for the data on deswelling, $D$ for very small volume changes is little affected by the phase separation. These values of $D$ also seem to converge to zero at $T_{\text {tr }}$, as in the case of Fig. 4(a); namely, the steep decrease of $D$ around $T_{\text {tr }}$ originates from the critical slowing-down.

By employing mode-coupling approximation, Onuki ${ }^{18}$ has shown that $D$ scales with the correlation length $(\xi)$ as

$$
D \sim \frac{1}{\xi^{2}},
$$

whereas Tanaka ${ }^{19}$ has applied the Kawasaki formula to $D$ : namely

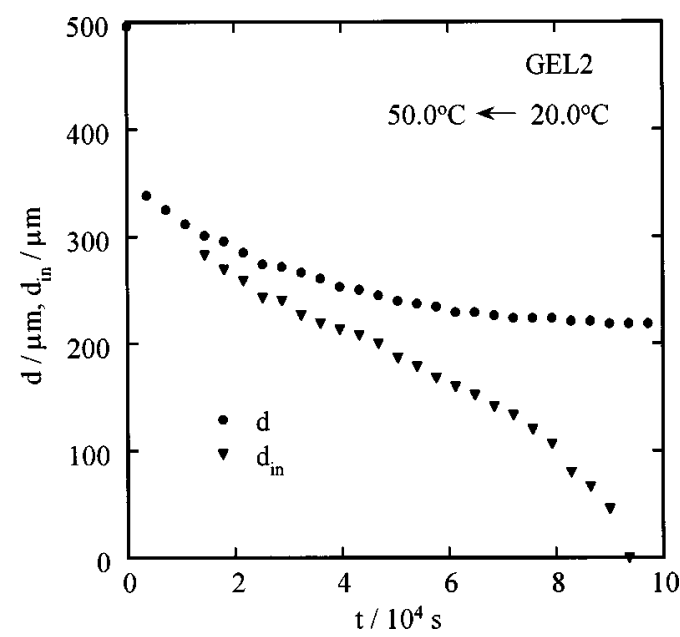

FIG. 2. Time $(t)$ dependence of the diameter of the gel $(d)$ and the diameter of the opaque region $\left(d_{\text {in }}\right)$ for GEL2. The $T$ jump (from $20.0{ }^{\circ} \mathrm{C}$ to $50.0^{\circ} \mathrm{C}$ ) is applied at $t=0$. At the short times, $d$ was identical to $d_{\text {in }}$, so that the three triangle symbols at $\mathrm{t}<4 \mathrm{~h}$ are eliminated from the figure.

$$
D \sim \frac{1}{\xi}
$$

Introducing a reduced temperature $(\epsilon)$ that represents the relative distance from the critical temperature $\left(T_{\mathrm{c}}\right)$ by ${ }^{20}$

$$
\epsilon=\frac{\left|T-T_{\mathrm{c}}\right|}{T_{\mathrm{c}}},
$$

near $T_{\mathrm{c}}, D$ is related to $\epsilon$ by

$$
D \sim \epsilon^{2 v}
$$

or

$$
D \sim \epsilon^{\nu},
$$



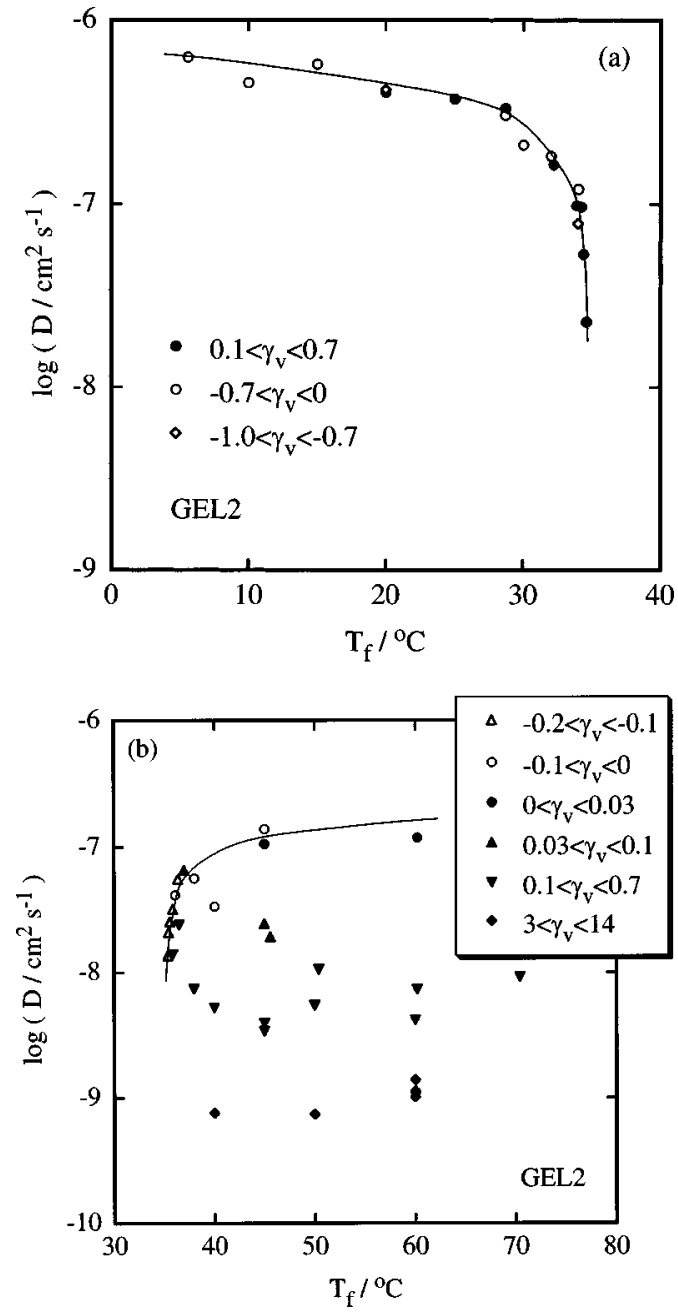

FIG. 4. Diffusion constant $(D)$ for GEL2 is plotted against the final temperature $\left(T_{\mathrm{f}}\right)$ for (a) $T_{\mathrm{f}}<35.0{ }^{\circ} \mathrm{C}$ and (b) $T_{\mathrm{f}}>35.0^{\circ} \mathrm{C}$. The data are classified into several categories depending on the value of swelling strain $\left(\gamma_{\mathrm{v}}\right)$. Open and filled symbols represent data on swelling and deswelling, respectively.

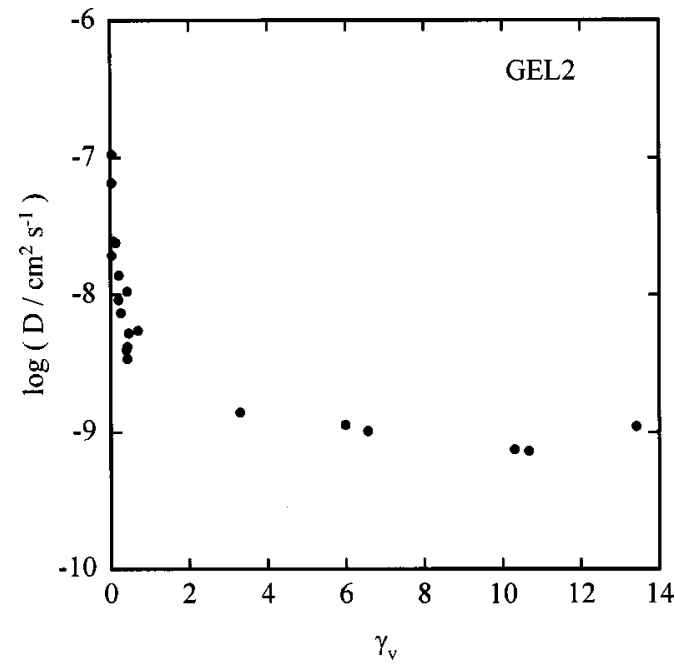

FIG. 5. The $\gamma_{\mathrm{v}}$ dependence of $D$ for GEL2 for the deswelling process where the phase separation occurs.

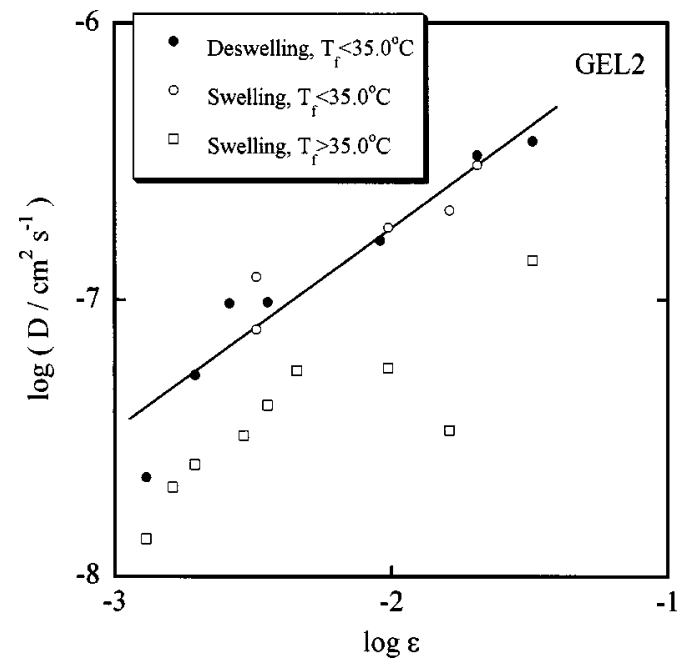

FIG. 6. The diffusion constant $D$ is double-logarithmically plotted against reduced temperature $(\epsilon)$ for GEL2. The data plotted are limited to those with $20^{\circ} \mathrm{C}<T_{\mathrm{f}}<50^{\circ} \mathrm{C}$. The solid line is the best fit to the data for $T_{\mathrm{f}}<T_{\text {tr }}$.

because $\xi \sim \epsilon^{-\nu}$. Here, $\nu$ is the critical exponent for $\xi^{20}$ The former scaling law corresponds to the scaling relation proposed by Onuki, the latter corresponding to the Tanaka relation. The critical temperature, $T_{\mathrm{c}}$, is $35.0^{\circ} \mathrm{C}, T_{\text {tr }}$ for GEL2. Figure 6 shows the semilogarithmic plots of $D$ against $\epsilon$ for GEL2. The data shown in Fig. 6 are limited to those in $20^{\circ} \mathrm{C}<T_{\mathrm{f}}<50^{\circ} \mathrm{C}$, and also the data on deswelling with $T_{\mathrm{f}}$ $>T_{\text {tr }}$ are eliminated, because $D$ depends strongly on $\gamma_{\mathrm{v}}$ as shown in Fig. 5. The solid line is the best fit to the data on both swelling and deswelling for $T_{\mathrm{f}}<T_{\mathrm{tr}}$.

On the basis of the Onuki relation, the value of $\nu$ was determined from the slope to be $0.37 \pm 0.03$. This value is smaller than the value either for the 3D Ising model $(\nu \sim 0.63)$ or for the mean field value $(\nu=0.5),{ }^{20}$ but is, if anything, closer to the mean-field value. On the other hand, $\nu=0.73 \pm 0.05$ for the PNIPA gel based on the Tanaka relation. In this case, the value is closer to the value for the $3 \mathrm{D}$ Ising than the mean field prediction. It has been reported that the universality class for the volume phase transition of the critical PNIPA gel belongs to the 3D Ising model..$^{20}$ Actually, according to the Tanaka relation the universality class of the transition can be considered to belong to the 3D Ising model. When the Onuki relation is valid, however, the universality class may be identical to that of the mean filed or classical theory. We think that the critical behavior for the polymer gel should obey the classical theory, because the existence of the polymer network in the gel must suppress the concentration fluctuation as Onuki has stated in Ref. 18; hence, the Onuki relation may be more appropriate for the description of the critical behavior of $D$. The exponent $\nu$ could not be determined for $T_{\mathrm{f}}>T_{\text {tr }}$, because the data points are scattered due to the difficulty in measuring small change in $d$. The value of the slope for the best fit line to the scattered data points for $T_{\mathrm{f}}>T_{\mathrm{tr}}$, however, appears to agree with that for $T_{\mathrm{f}}<T_{\mathrm{tr}}$.

Figures 7 and 8 show the $T_{\mathrm{f}}$-dependence of $D$ for GEL1 and GEL3, respectively. Table I contains the detailed data for the gels. The diffusion constant $D$ plotted in these figures are limited for the relatively small volume change processes. 


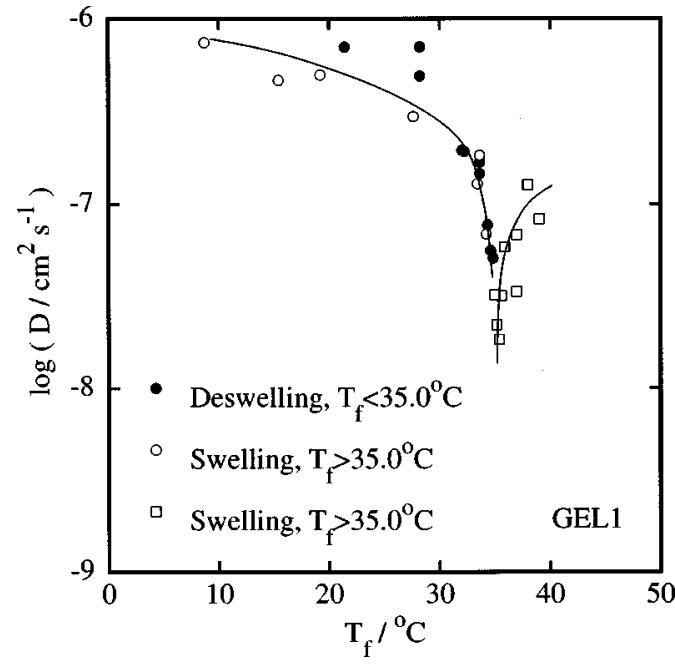

FIG. 7. The diffusion constant $D$ for GEL1 is plotted against $T_{\mathrm{f}}$. Open and filled symbols represent data on swelling and deswelling, respectively.

The data obtained from the deswelling processes for $T_{\mathrm{f}}$ $>T_{\text {tr }}$ was also eliminated, because the effects of homogenization after phase separation exist on the deswelling kinetics. The values of $D$ for both GEL1 and GEL3 decrease rapidly as $T_{\mathrm{f}}$ approaches $35.0^{\circ} \mathrm{C}$, just as observed for GEL2. The $T_{\mathrm{f}}$-dependence curves of $D$ for the three gels seem to be identical to one another both in shape and magnitude. Theoretically speaking, the values of $D$ for GEL1 and GEL3 do not converge to zero even at the transition temperature $T_{\text {tr }}$ $\left(=35.0^{\circ} \mathrm{C}\right)$, because the gels are off-critical. GEL1 shows no phase transition, so that the characteristic time $\tau$ for volume change does not diverge even at $T_{\text {tr }}$, giving a finite value of $D$ even at the transition temperature. In the case of GEL3 showing the first-order phase transition, $\tau$ diverges at the spinodal temperature $\left(T_{\mathrm{s}}\right)$, which is slightly different from the transition temperature, and $D$ converges to zero at $T_{\mathrm{s}}$. This means that the $T_{\mathrm{f}}$-dependence curve of $D$ for GEL3 must have a cusp at $T_{\text {tr }}$, and $D$ for GEL3 must also have a nonzero value at $T_{\mathrm{c}}$. In Figs. 9 and 10,D for GEL1 and

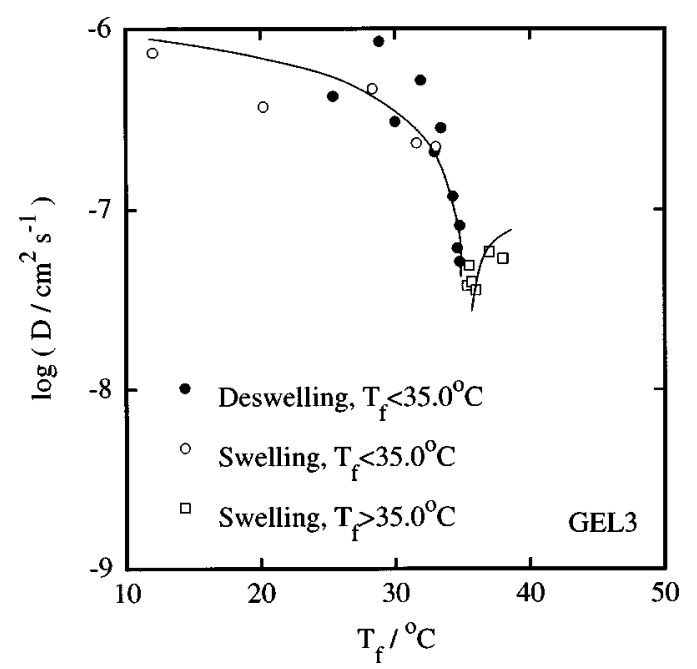

FIG. 8. The diffusion constant $D$ for GEL3 is plotted against $T_{\mathrm{f}}$. Open and filled symbols represent data on swelling and deswelling, respectively.

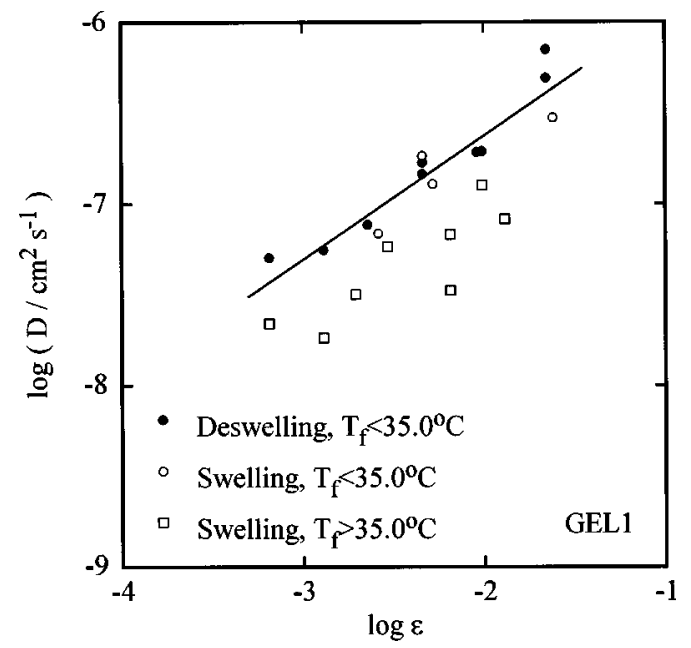

FIG. 9. The diffusion constant $D$ is double-logarithmically plotted against $\epsilon$ for GEL1. The data plotted are limited to those with $20.0^{\circ} \mathrm{C}<T_{\mathrm{f}}$ $<50.0^{\circ} \mathrm{C}$. The solid line is the best fit to the data for $T_{\mathrm{f}}<T_{\mathrm{tr}}$.

GEL3 is, respectively, plotted against $\epsilon$. Here, $T_{\mathrm{tr}}=T_{\mathrm{c}}$ was assumed. The data plotted are limited to those in $20.0^{\circ} \mathrm{C}$ $<T_{\mathrm{f}}<50.0^{\circ} \mathrm{C}$. The value of $\nu$ obtained from the slope of the best fit line to the data points is $0.34 \pm 0.03$ both for GEL1 and GEL3 when the Onuki relation is used. This value is almost identical to that for GEL2, resulting from very small values of $D$ at $T_{\mathrm{c}}$ for GEL1 and GEL3, although the theory of the critical phenomena predicts $\nu=0$ for the gels.

\section{CONCLUSION}

Swelling and deswelling kinetics was investigated for the three types of the cylindrical PNIPA gels by the $T$-jump method. In the deswelling processes at $T_{\mathrm{f}}>35.0^{\circ} \mathrm{C}$, the phase separation was observed in the gels. The homogenization after the phase separation slowed down the deswelling process, resulting in the small value of $D$. The value of $D$ obtained without the phase separation decreased rapidly as $T_{\mathrm{f}}$ approached the transition temperature $T_{\mathrm{tr}}$. For the critical gel

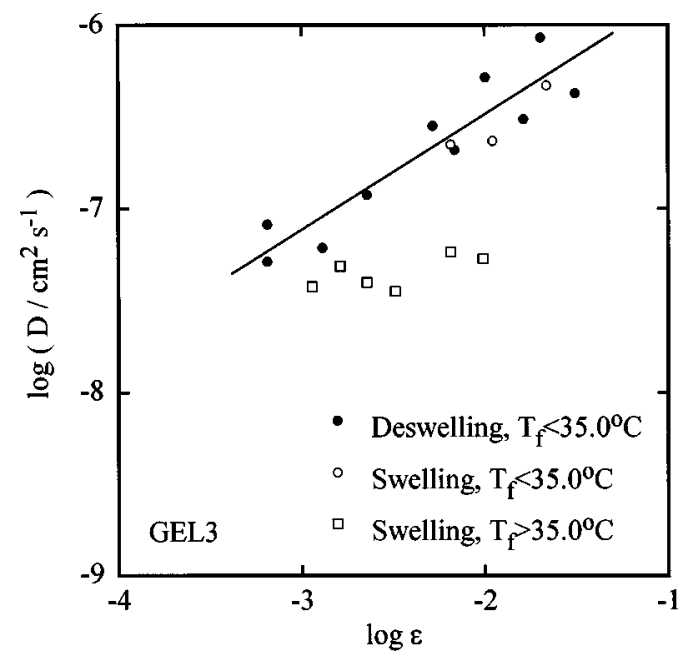

FIG. 10. The diffusion constant $D$ is double-logarithmically plotted against $\epsilon$ for GEL3. The data plotted are limited to those with $20.0^{\circ} \mathrm{C}<T_{\mathrm{f}}$ $<50.0^{\circ} \mathrm{C}$. The solid line is the best fit to the data for $T_{\mathrm{f}}<T_{\mathrm{tr}}$. 
(GEL2 in this study), this rapid decrease of $D$ near $T_{\text {tr }}$ suggests the emergence of the critical slowing-down. The value of the critical exponent $\nu$ for GEL2 was determined to be $0.37 \pm 0.03$, when a mode-coupling approximation by Onuki was employed. This indicates that the universality class for the volume phase transition of the critical PNIPA gel belongs to the class for the classical theory. According to the theory of the critical phenomena, it can be expected that $D$ for the off-critical gels (GEL1 and GEL3) does not converge to zero at $T_{\mathrm{c}}$. The $T_{\mathrm{f}}$-dependence curves of $D$ for off-critical gels were similar in shape with that for the critical gel; namely, the off-critical gels showed pseudo-critical behavior on the $D-T_{\mathrm{f}}$ curves. This means that the bottom of the $D-T_{\mathrm{f}}$ curves for the off-critical gels is very deep.

${ }^{1}$ T. Tanaka and D. Fillmore, J. Chem. Phys. 70, 1214 (1979).

${ }^{2}$ A. Peters and S. J. Candau, Macromolecules 19, 1952 (1986).

${ }^{3}$ A. Peters and S. J. Candau, Macromolecules 21, 2278 (1988).

${ }^{4}$ Y. Li and T. Tanaka, J. Chem. Phys. 92, 1365 (1990).
${ }^{5}$ C. Wang, Y. Li, and Z. Hu, Macromolecules 30, 4727 (1997).

${ }^{6}$ T. Tanaka, L. O. Hocker, and G. B. Benedek, J. Chem. Phys. 59, 5151 (1973).

${ }^{7}$ E. S. Matsuo and T. Tanaka, J. Chem. Phys. 89, 1695 (1988).

${ }^{8}$ Y. Li and T. Tanaka, J. Chem. Phys. 90, 5161 (1989).

${ }^{9}$ M. Tokita and T. Tanaka, Science 253, 1121 (1991).

${ }^{10}$ M. Shibayama, T. Tanaka, and C. C. Han, J. Chem. Phys. 97, 6829 (1992).

${ }^{11}$ T. Tanaka, E. Sato, Y. Hirokawa, S. Hirotsu, and J. Peetermans, Phys. Rev. Lett. 55, 2455 (1985).

${ }^{12}$ T. Takigawa, T. Yamawaki, K. Takahashi, and T. Masuda, Polym. J. (Tokyo, Jpn.) 31, 595 (1999).

${ }^{13} \mathrm{H}$. Hirose and M. Shibayama, Macromolecules 31, 5336 (1998).

${ }^{14}$ M. Shibayama and K. Nagai, Macromolecules 32, 7461 (1999).

${ }^{15}$ A. Suzuki, S. Yoshikawa, and G. Bai, J. Chem. Phys. 111, 360 (1999).

${ }^{16}$ G. Bai and A. Suzuki, J. Chem. Phys. 111, 10338 (1999).

${ }^{17}$ T. Okajima, I. Harada, K. Nisho, and S. Hirotsu, J. Chem. Phys. 116, 9068 (2002).

${ }^{18}$ A. Onuki, Adv. Polym. Sci. 109, 63 (1993).

${ }^{19}$ T. Tanaka, Phys. Rev. A 17, 763 (1978).

${ }^{20} \mathrm{H}$. E. Stanley, Introduction to phase transitions and critical phenomena (Clarendon, Oxford, 1971). 